LAWRENCE LIVERMORE NATIONAL LABORATORY

\title{
Instability Driven by Sheath Boundary Conditions and Limited to Divertor Legs
}

D. D. Ryutov and R. H. Cohen

September 3, 2003

Plasma Edge Theory Conference, San Diego, California, September 3-5, 2003 


\section{DISCLAIMER}

This document was prepared as an account of work sponsored by an agency of the United States Government. Neither the United States Government nor the University of California nor any of their employees, makes any warranty, express or implied, or assumes any legal liability or responsibility for the accuracy, completeness, or usefulness of any information, apparatus, product, or process disclosed, or represents that its use would not infringe privately owned rights. Reference herein to any specific commercial product, process, or service by trade name, trademark, manufacturer, or otherwise, does not necessarily constitute or imply its endorsement, recommendation, or favoring by the United States Government or the University of California. The views and opinions of authors expressed herein do not necessarily state or reflect those of the United States Government or the University of California, and shall not be used for advertising or product endorsement purposes.

This work was performed under the auspices of the U. S. Department of Energy by the University of California, Lawrence Livermore National Laboratory under Contract No. W-7405-Eng-48. 


\title{
Instability Driven by Sheath Boundary Conditions and Limited to Divertor Legs
}

\author{
D.D. Ryutov, R.H. Cohen \\ Lawrence Livermore National Laboratory, Livermore, CA, 94551 \\ e-mail address of submitting author: ryutov1@1lnl.gov
}

\begin{abstract}
An instability driven by an electron temperature gradient in combination with sheath boundary conditions at a divertor plate is considered. It is shown that there exists a mode localized between the divertor plate and the x point. Further propagation of the mode is terminated by a strong shear near the x point. A "heuristic" boundary condition at the control surface situated somewhat below the $\mathrm{x}$ point is suggested. The mode manifests a strong dependence on the radial tilt of the divertor plate, thereby providing some degree of control over the plasma transport in the divertor leg. Estimates of the diffusion coefficient show that it may reach the Bohm value.
\end{abstract}

\section{I.Introduction}

A very fast instability driven by sheath boundary conditions was predicted to exist on the open field lines of fusion devices [1,2]. Its presence is generally not related to the sign of the field-line curvature, although it becomes somewhat faster in the case of unfavorable curvature. It was pointed out in Ref. [3] that, in divertor geometry, the most unstable perturbations (perturbations with large toroidal mode numbers) cannot penetrate beyond the $\mathrm{X}$ point because of strong shearing that leads to a dramatic increase of the radial wave number and corresponding rapid damping of the perturbations just above the $\mathrm{X}$ point. Depending on the specifics of the divertor design, low-toroidal-mode-number perturbations may survive the transition through the $X$ point [4]. Here we consider a situation where this is not the case and divertor legs are decisively disconnected from the region above the $\mathrm{X}$ point.

This opens up a possibility that the instability will be confined to the region between the divertor plate and the $\mathrm{X}$ point, and will not not penetrate to the upper part of the scrape-off-layer (SOL). The reverse effect, an effective decoupling (from the divertor legs) of the instability above the X point, has been demonstrated in Ref. [5], where the corresponding mode was called "resistive X-point mode."

In a simple heuristic model that we will pursue in this paper, the role of the Xpoint is described by a boundary condition imposed on the perturbations on a "control surface" somewhat below the $\mathrm{x}$ point. This boundary condition stems from general qualitative considerations and seems to be quite robust. We then consider perturbations similar to those described in Refs. [1,2], with the main driving term proportional to the gradient of the electron temperature. Boundary conditions on the divertor plate are similar to those imposed in Ref. [6]. 
In this paper, we analyze a low-recycling divertor, with plasma parameters almost constant along a field line. The plasma is assumed to flow to the divertor plate with a velocity which somewhat exceeds the sound speed. Under such conditions, we find a robust and rapidly growing mode that may affect the plasma transport in the divertor legs without affecting it in the scrape off layer above the $\mathrm{X}$ point. We find that the tilt of the divertor plate with respect to the poloidal magnetic field (i.e., a tilt associated with a rotation of the plate around the toroidal direction; we call it the "radial tilt") provides a substantial degree of control over the instability. We provide estimates of the transport coefficients within a mixing-length approximation.

To put our study in some prospective, we briefly mention earlier papers related to the sheath-driven instability. The notion that the sheath boundary conditions may affect the plasma stability can be traced back to the seminal papers by Kunkel and Guillory [7] and Kadomtsev [8]. In Ref. [1] where the instability was first clearly identified and linked to SOL problems, finite-beta effects were included. In Ref. [2], effects of finite ion Larmor radius were added to the analysis. In Ref. [9], finite plasma resistivity was included and detailed analysis of the instability was carried out for tokamak SOL conditions. In Ref. [6], the radial tilt of the divertor plate was added to the analysis and found to be important. In Ref. [3], the aforementioned dramatic role of the X-point shear in the decoupling the sheath effects from the SOL above the X point was first discussed. In Ref. [4], it was suggested that low toroidal mode numbers may, in fact, be not very sensitive to this effect. In Ref. [10], the interference of the sheath-driven mode and velocity-shear modes was discussed. Later on, a more detailed numerical analysis of the same problem was carried out in Ref. [11]. In Ref. [12], effect of collisions with neutrals was added to the analysis. In Ref. [13], effects of parallel shear were studied. Finally, in Ref. [5] an extensive analysis of the modes existing in the main part of the SOL and their interaction with the x-point region was performed. An eikonal technique was used to consider perturbations in the x-point region and detailed numerical results of the mode structure were presented. It was found that, in a number of cases, perturbations do not penetrate below the $\mathrm{x}$-point.

\section{The Model}

For this initial analysis we limit ourselves to a simplest model where only the most essential features of the full problem are taken into consideration. We use a slab model of the section of the divertor leg between the divertor plate and a "control surface" situated somewhat below the x-point (Fig. 1). The exact location of this surface is not very important, especially in the case of "long" divertor legs. We use Cartesian coordinates with the axes $x, y$, and $z$ being analogs of radial, poloidal and toroidal coordinates, respectively. The origin is situated on the control plane. We allow for the possibility that the normal to the plate is not collinear with axis $y$ (the aforementioned "radial tilt" characterized by angle $\alpha$ ). The distance between the control plane and the divertor plate along the axis y (i.e., in the poloidal direction) is $l(y)$.

The plasma is assumed to enter the divertor leg through the control surface at some speed $u$. The unperturbed plasma parameters are assumed not to vary between the control surface and the divertor plate. This assumption corresponds to a situation of a 
relatively low plasma and neutral density, where the electron temperature is uniform along field lines and where ionization and charge exchange are negligible.

The most important source of the instability described in [1,2] is the radial variation of the electron temperature. Accordingly, we assume that the density and the ion temperature do not depend on the coordinate $x$, and the only plasma parameter varying in the $x$ direction is the electron temperature $T_{e}(x)$. (It will soon become clear that neglecting gradients of density and ion temperature is justified if they have the same or larger scale-length as the electron temperature).

As the electron temperature varies along the coordinate $x$, so does the plasma potential. In other words, a radial $(x)$ unperturbed electric field $E_{0}$ is present in the plasma. It is roughly equal to

$$
E_{0}=-\frac{\Lambda T_{e}^{\prime}}{e}
$$

where prime here and below means differentiation over $\mathrm{x}$, and $\Lambda$ is a familiar logarithmic factor $\sim 2-3$. This electric field creates a drift, whose poloidal velocity is

$$
\mathrm{v}_{D}=\frac{c E_{0} B_{z}}{B^{2}}
$$

We assume that the poloidal drift velocity is smaller than the projected poloidal component of the plasma flow along the field lines,

$$
\mathrm{v}_{\mathrm{D}}<\frac{B_{y}}{B} u
$$

We consider frequencies well below the ion gyrofrequency and wavenumbers well below the inverse ion gyroradius. Then the plasma cross-field velocity is determined by the equation

$$
\delta \mathbf{v}_{\perp}=c \frac{\mathbf{B} \times \nabla \delta \varphi}{B^{2}}
$$

The instability will be shown to have a growth rate much higher than the plasma transit time over the connection length $L=l B_{z} / B_{y}$; accordingly, the plasma distribution along the flux tube does not change within the instability growth time. This means that perturbations of plasma parameters are purely convective, so that the electron temperature perturbation obeys an equation

$$
\frac{\partial \delta T_{e}}{\partial t}+\mathbf{v}_{D} \cdot \nabla \delta T_{e}=-\delta \mathrm{v}_{x} T_{e}^{\prime}
$$

Perturbations of the plasma density and ion temperature are zero, as the corresponding unperturbed quantities are uniform. The plasma momentum can then be written as:

$$
\rho \delta\left(\frac{d \delta \mathbf{v}}{d t}\right)=-n \nabla \delta T_{e}+\frac{\delta \mathbf{j} \times \mathbf{B}}{c}
$$

After that, we consider perturbations with cross-field scale length much smaller then the SOL width $\Delta$ and therefore use a local WKB analysis, with perturbations of the form $\exp (-i \omega+i \mathbf{q} \cdot \mathbf{r})$, with the wave vector perpendicular to the magnetic field, $(\mathbf{q} \cdot \mathbf{B})=0$. It is convenient to present the vector $\boldsymbol{q}$ as a sum of the two components: a radial component $\boldsymbol{q}_{r}$ and the component $\boldsymbol{k}=\boldsymbol{q}-\boldsymbol{q}_{r}$ perpendicular to both the magnetic field and the axis $x$ (Fig.1). For such perturbations Eqs. (4)-(6) yield:

$$
\delta \mathbf{j}_{\perp}=-\frac{c}{B^{2}}\left[\Omega \rho c \mathbf{q}+\frac{i k c n}{\Omega B}(\mathbf{B} \times \mathbf{q}) T_{e}^{\prime}\right] \delta \varphi
$$


where

$$
\Omega=\omega-\mathbf{k} \cdot \mathbf{v}_{D}
$$

At this point, we use the current continuity equation which allows us to find the parallel current from the equation $\partial j_{\|} / \partial s=-i \mathbf{q} \cdot \delta \mathbf{j}_{\perp}$ where $s$ is a coordinate along the field line. Integration of this equation from the upper control surface to the divertor plate with account for Eq. (7) yields:

$$
\delta j_{\|}^{(\text {plate })}-\delta j_{\|}^{(x-p o \text { int })}=\frac{i c^{2} k^{2} L M n \Omega}{B^{2}} \delta \varphi
$$

To close the set of equations and to arrive at the dispersion relation, we need to impose boundary conditions relating perturbations of the current and of the potential at the end surfaces. We discuss this issue in the next two sections.

\section{A heuristic model for the "surface conductivity" of the control surface.}

Consider the plasma potential perturbations at the "control surface." In Sec.II we have described plasma motions that these perturbations would induce in the divertor leg. Here we discuss the reaction to their presence of the area above the control surface, in the zone near and above the x-point. As was shown in Ref. [3], a flux-tube with initially circular cross-section, when passing near the x-point, squeezes very strongly and soon becomes thinner than the ion gyroradius. As was shown in Ref. [3] and later discussed in more detail in Ref. [14] (see Eqs. (A.3.19), (A.3.20) of that reference), this happens very quickly. The situation at this stage is schematically shown on Fig. 2: the ion orbits are larger than the scale at which potential varies. The effective wave number becomes equal to

$$
K(s)=q E(s),
$$

where $E$ is a so called elongation of initially circular flux tube (defined as the ratio of the major semi-axis of the squeezed flux tube to its initial radius at the control surface). In this case, the ion average cross-field velocity is much less than $\mathrm{v}_{\mathrm{D}}$.

At the same time, we assume that the wave number $K$ is still much less than the inverse electron gyroradius: as it turns out, the potential perturbations decay close enough to the control surface, where the elongation still remains moderate (see below). Under such circumstances, the presence of the perpendicular electric field $i K \delta \varphi$ leads to electron cross-field current

$$
\delta j_{\perp}=\sigma_{\perp}(-i K \delta \varphi)=-i k \sigma_{\perp} E(s) \delta \varphi
$$

where

$$
\sigma_{\perp}=\frac{\omega_{p e}^{2} \nu}{4 \pi \omega_{C e}^{2}},
$$

where and $\omega_{p e}$ and $\omega_{C e}$ are electron plasma and electron cyclotron frequency, respectively, and $v$ is an electron collision frequency.

The divergence of the perpendicular current is balanced by the parallel current which varies with the distance s:

On the other hand, one has

$$
\frac{\partial \delta j_{\|}}{\partial s}=-i K \delta j_{\perp}=-k^{2} E(s) \sigma_{\perp} \delta \varphi
$$




$$
\delta j_{\|}=-\sigma_{\|} \frac{\partial \delta \varphi}{\partial s}
$$

where

$$
\sigma_{\|}=\frac{\omega_{p e}^{2}}{4 \pi v}
$$

Assuming that $K^{2}>d K / d s$, which holds by a large margin, one finds from Eqs. (13), (14) that

$$
\delta \varphi \approx \delta \varphi_{0} \exp (-I)
$$

where

$$
I=k \sqrt{\frac{\sigma_{\perp}}{\sigma_{\perp}}} \int_{0}^{s} E\left(s^{\prime}\right) d s^{\prime}=\frac{k v}{\omega_{C e}} \int_{0}^{s} E\left(s^{\prime}\right) d s^{\prime}
$$

Now we find the relationship between the potential and current perturbation at the control plane:

$$
\delta j_{\| 0}=-\left.\sigma_{\|} \frac{\partial \varphi}{\partial s}\right|_{s=0}=k \sigma_{e f f} \delta \varphi_{0}
$$

where $\sigma_{\text {eff }}$ is defined as

$$
\sigma_{e f f}=\sqrt{\sigma_{\|} \sigma_{\perp}}=\frac{\omega_{p e}^{2}}{4 \pi \omega_{C e}}
$$

Remarkably, this result does not contain any details related to the particular formulation of the problem and is quite robust in this regard.

For the exponential $s$-dependence of $E$ (see [3], [14]), $E \approx \exp \left(s / L^{*}\right)$, where $L^{*}$ is a characteristic length (typically, 5-10 m), one has

$$
I=\frac{k v}{\omega_{C e}} \int_{0}^{s} E\left(s^{\prime}\right) d s^{\prime}=\frac{k \rho_{C e} L^{*}}{\lambda}(E(s)-1)
$$

From this equation one concludes that, at the distance where the potential perturbation becomes exponentially small, one has $K \rho_{C e} \sim \lambda / L^{*}$. As the r.h.s.is typically much less than 1, the applicability condition (that $K \rho_{C e}<1$ ) is satisfied.

Still given possible uncertainties of a real problem, we introduce some phenomenological coefficient $G$ to the expression for $\sigma_{e f f}$ i.e., we write

$$
\sigma_{e f f}=\frac{G \omega_{p e}^{2}}{4 \pi \omega_{C e}}
$$

and study the sensitivity of the results to the coefficient $G$ in Sec. V of the paper.

The current expressed by Eq. (18) is exactly the current $\delta j_{\|}^{x-p o \text { int }}$ that enters Eq. (9). This equation allows us, therefore, to find the parallel current at the divertor plate,

$$
\delta j_{\|}^{(d i v)}=\left(\frac{i c^{2} k^{2} L M n \Omega}{B^{2}}+k \sigma_{e f f}\right) \delta \varphi
$$

Using also Eq. (7), one can now obtain an expression for the normal component of the current flowing from the plasma to the divertor plate: 


$$
\begin{aligned}
& \delta j_{n}^{(d i v)}=\left(n_{y} \delta j_{\|}+\mathbf{n} \cdot \delta \mathbf{j}_{\perp}\right)^{(d i v)}=i \delta \varphi \frac{c^{2} q^{2} n}{B^{2}} \times \\
& {\left[\left(L M \Omega-i \frac{B^{2} \sigma_{e f f}}{n c^{2} q}\right) \frac{B_{y}}{B} \sin \alpha+\frac{T_{e}^{\prime}}{\Omega} \frac{k^{2}}{q^{2}}\left(\cos \alpha+\frac{q_{r} B_{z}}{k B} \sin \alpha\right)\right]}
\end{aligned}
$$

We have neglected the contribution of the first term in Eq. (7) which is $k L$ times smaller than the contribution from $\delta j_{\|}$. Further simplifications are related to the smallness of $B_{y}$ that allows one to set $B_{z} \approx B$, and assumption that $q_{r}<<k$ (which, as was shown in Refs. [2] and [6], corresponds to the largest growth rate for the instability that we are considering). This yields:

$$
\delta j_{n}^{(d i v)}=i \delta \varphi \frac{c^{2} k^{2} n}{B^{2}}\left[\left(L M \Omega-i \frac{B^{2} \sigma_{e f f}}{n c^{2} k}\right) \frac{B_{y}}{B} \sin \alpha+\frac{T_{e}^{\prime}}{\Omega} \cos \alpha\right]
$$

\section{Sheath boundary condition at the divertor plate}

The sheath boundary condition suitable for this problem was derived in Ref. [6], in a linearized form. It was later derived in a more general form (Eq. (19) in Ref. [15]), without an assumption of the smallness of the perturbations. Note that in our present notation, the angle $\alpha$ used in reference [15] should be replaced by $\alpha\left(\mathrm{B}_{\mathrm{y}} / \mathrm{B}_{\mathrm{z}}\right)$. Linearizing Eq. (19) of Ref. [15], or, equivalently, using Eq. (37) of Ref. [6] we obtain:

$$
\delta j_{n}^{\text {(plate })}=\delta \varphi \sin \alpha \frac{B_{y}}{B} e n u\left[-\frac{i c k}{u B_{y}} \cot \alpha+\left(\Lambda+\frac{1}{2}\right) \frac{k c}{\Omega B} \frac{T_{e}^{\prime}}{T_{e}}+\frac{e}{T_{e}}\right]
$$

Note that there is a sizeable logarithmic factor $\Lambda$ in the term arising from the electron temperature perturbation - it demonstrates that even if we allowed for the presence of unperturbed gradients of the plasma density or the ion temperature and thereby make perturbations of these latter quantities non-zero, the $T_{e}^{\prime}$ drive is still more prominent.

\section{Analysis of the dispersion relation}

Equating expressions (24) and (25), one finds the following dispersion relation:

where

$$
\Omega^{2}+\Omega\left(i \Omega_{1}+\Omega_{2}+i \Omega_{3}\right)-i \Gamma_{1}^{2}-\Gamma_{2}^{2}=0
$$

$$
\begin{aligned}
& \Omega_{1}=\frac{\omega_{C i}^{2} M u}{L k^{2} T_{e}}, \quad \Omega_{2}=\frac{\omega_{C i}}{k l} \cot \alpha, \Omega_{3}=\frac{\omega_{C i} G}{k L}, \\
& \Gamma_{1}^{2}=\left(\Lambda+\frac{1}{2}\right) \frac{\omega_{C i} u}{k L} \frac{T_{e}^{\prime}}{T_{e}}, \Gamma_{2}^{2}=\frac{T_{e}^{\prime}}{M L} \cot \alpha
\end{aligned}
$$

The meaning of various terms in the dispersion relation (26) is as follows: The first term represents the inertia of the flute. Frequency $\Omega_{1}$ describes the Kunkel-Guillory sheath resistivity. Frequency $\Omega_{2}$ describes correction to the sheath current-voltage characteristic associated with the tilt of the plate. Frequency $\Omega_{3}$ describes the effect of the X-point resistivity suggested in this paper. The term $\Gamma_{1}^{2}$, together with the frequency $\Omega_{1}$, describes 
the instability of Refs. (1), (2). The term $\Gamma_{2}^{2}$ describes an instability associated with the work performed by the electron pressure when the flute is sliding over the surface of the tilted plate, so that the flute length varies. This is possible because the sheath is essentially perfectly reflecting for the electron gas. This instability in its "pure" form (i.e., with all the terms in (26), except for the first and the last are dropped) is essentially identical to the flute instability, as shown in Ref. [16].

We now switch to convenient dimensionless variables first introduced in Ref. [6]:

$$
\hat{\Omega}=\Omega / \Omega_{0} ; \hat{k}=k / k_{0}
$$

where

$$
\begin{aligned}
& k_{0}=\frac{\omega_{C i}}{u}\left(\frac{1}{\Lambda+\frac{1}{2}} \frac{\Delta}{L}\right)^{1 / 3}\left(\frac{T_{e}}{M u^{2}}\right)^{-2 / 3}, \\
& \Omega_{0}=\frac{u}{L}\left(\frac{1}{\Lambda+\frac{1}{2}} \frac{\Delta}{L}\right)^{-2 / 3}\left(\frac{T_{e}}{M u^{2}}\right)^{1 / 3} . \\
& \Delta=T_{e} /\left|T_{e}^{\prime}\right|
\end{aligned}
$$

The dispersion relation then becomes

$$
\hat{\Omega}^{2}+\hat{\Omega}\left\{\frac{i}{\hat{k}^{2}}+\frac{C}{\hat{k}}\left[\left(\Lambda+\frac{1}{2}\right) \cot \alpha+\frac{i G l}{L}\right]\right\}+\frac{i}{\hat{k}}+C \cot \alpha=0 .
$$

We assume that $T_{e}^{\prime}<0$. In the opposite case (as it would be in the private flux region), the sign of the last two terms should be changed to "-". All the characteristics of the system (except for geometrical parameters) are folded into a single dimensionless parameter

$$
C=\frac{1}{\Lambda+\frac{1}{2}} \frac{L}{l}\left(\frac{1}{\Lambda+\frac{1}{2}} \frac{\Delta}{L}\right)^{1 / 3}\left(\frac{T_{e}}{M u^{2}}\right)^{1 / 3}
$$

As a numerical example, consider a medium-scale tokamak, with the following parameters of the divertor: $B=1 \mathrm{~T}, B_{y}=0.1 \mathrm{~T}, l=50 \mathrm{~cm}, L=500 \mathrm{~cm}, \Delta=5 \mathrm{~cm}, T_{e}=30 \mathrm{eV}$, and $M u^{2}=2 T_{e}$, hydrogen plasma. Taking $L$ to be 2.5 , one finds that, for these parameters, $k_{0}=3$ $\mathrm{cm}^{-1}$, whereas the ion gyroradius $\rho_{i}$, evaluated for the electron temperature, is $0.08 \mathrm{~cm}$. In other words, the applicability conditions for our analysis,

$$
\rho_{i}<k_{0}^{-1}<\Delta,
$$

is satisfied. The characteristic frequency $\Omega_{0}$ is much higher than the inverse transit time, $\Omega_{0} \sim 30(u / L) \sim 5 \times 10^{5} \mathrm{~s}^{-1}$. It is, however, more than two orders of magnitude less than the ion gyro-frequency. The value of $C$ is $C=0.4$.

For the case where radial tilt is absent $\left(\alpha=90^{\circ}\right)$, the plot of the growth rate vs. the wave number for several values of the parameter $G$ is shown in Fig. 3. One sees that the results are not very sensitive to the possible uncertainty of the numerical value of $G$.

In Fig. 4, we plot the growth rates for various values of the tilt. One sees that the "unfavorable " $(\alpha>0)$ tilt leads to a substantial increase of the growth rate. At large $k$ 's the instability becomes similar to a hydrodynamic flute instability driven by the unfavorable 
curvature. In our case, the drive is, however, different: it is related to the liberation of the electron energy in the course of the flute sliding along the tilted surface of the divertor plate. For "favorable" tilt, the instability gets suppressed compared to the $\alpha=90^{\circ}$ case.

\section{Discussion}

In the present paper, we suggested a model that describes the effect of an x-point on the perturbations below this point in terms of a simple boundary condition. The formulation of this condition is based on the observation that, due to the strong x-point shear, the radial wave number of a flute-like perturbation becomes large compared to the inverse ion gyroradius, and a cross-field electron conductivity terminates the perturbation near the $\mathrm{x}$ point. For the plasma in the divertor leg, the boundary condition relates potential perturbations on some "control surface" below the x-point to the parallel currents closing in the x-point vicinity. This relation can be expressed in terms of some "effective electrical conductivity" which does not depend on any plasma parameters but the density (Eq. (19)). Still, we call this model "heuristic", because it is not based on a detailed analysis of the potential perturbations in the x-point zone. So, we introduce a numerical coefficient $G$ which accounts for this uncertainty and make a parametric study of its effect on the instability. We find this effect insignificant.

These observations allow one to study the instability in the divertor leg separately from the processes going on beyond the x-pint.. With the boundary condition of the aforementioned type on the control surface, and a sheath boundary condition on the divertor plane, we analyze the instability driven by the electron temperature gradient. Its growth rate turns out to be strongly affected by the radial tilt of the divertor plate. For one sign of the tilt, the instability increases in the common flux region and decreases in the private flux region and vice versa for the opposite sign. This provides a potentially interesting tool for controlling transport in divertors.

The characteristic diffusion coefficient, which can be estimated in the mixing length approximation, is

$$
D_{0} \equiv \frac{\Omega_{0}}{k_{0}^{2}}=\frac{\left(T_{e} / M\right)^{3 / 2}}{\Delta \omega_{C i}^{2}}\left(\frac{L}{\Delta}\right)^{1 / 3}\left(\Lambda+\frac{1}{2}\right)^{4 / 3}\left(\frac{T_{e}}{M u^{2}}\right)^{1 / 6}
$$

It scales as $1 / B^{2}$ but is much greater than the classical value. For the numerical example mentioned in Sec.V, it is close to the Bohm value. It becomes a few times greater than Bohm for the proper radial tilt of the divertor plate. So, potentially, for a divertor with long legs, this instability may provide additional opportunities for spreading the plasma over a large area of the divertor plate and reducing heat loads.

\section{Acknowledgment}

This work was performed under the auspices of the U.S. Department of Energy by University of California Lawrence Livermore National Laboratory under contract \# W7405-Eng-48.

\section{References}

1. H.Berk, D. D. Ryutov, Yu. A.Tsidulko JETP Lett., 52, 23 (1990). 
2. H.Berk, D. D. Ryutov, Yu. A.Tsidulko. Phys.Fluids, B3, 1346 (1991).

3. D.Farina, R.Pozzoli, D. Ryutov. Nuclear Fusion, 33, 1315 (1993).

4. R.H. Cohen, D.D. Ryutov. Contrib. Plasma Phys., 36, 161 (1996).

5. J.R. Myra, D.A. D’Ippolito, X.Q. Xu, R.H. Cohen. Phys. Plasmas, 7, 4622 (2000).

6. D.Farina, R.Pozzoli, D. Ryutov. Plasma Phys. Contr. Fusion, 35, 1271 (1993).

7. W. Kunkel, J. Guillory. In: "Phenomena in Ionized Gases" (Proc. \& th Conf. Belgrade, 1965) Vol. 2, p. 702, Belgrade, 1966

8. B.B. Kadomtsev, ibid, p. 610.

9. H.L. Berk, R.H. Cohen, D.D. Ryutov,Y.A. Tsidulko, X.Q. Xu. Nuclear Fusion, 33, 263 (1993).

10. K.Lotov, D. Ryutov, J. Weiland. Physica Scripta, 50, 153 (1994).

11. D.R. McCarthy, P.J. Catto, S.I. Krasheninnikov. Phys. Plasmas, 8, 750 (2001).

12. X.Q. Xu, R.H. Cohen RH. Plasma Phys. Contr. Fusion, 35, 1071 (1993).

13. Y.A. Tsidulko, H.L. Berk, R.H. Cohen. Physics of Plasmas. 1, 1199 (1994)

14. D.D. Ryutov, R.H. Cohen, P. Helander. Plasma Phys. Contr. Fusion, 43, 1399, (2001).

15. R.H.Cohen, D.D. Ryutov. Phys. Plasmas, 2, 2011 (1995).

16. D.Farina, R.Pozzoli, D.D. Ryutov. Phys.Fluids, v.B5, p.4055 (1993). 

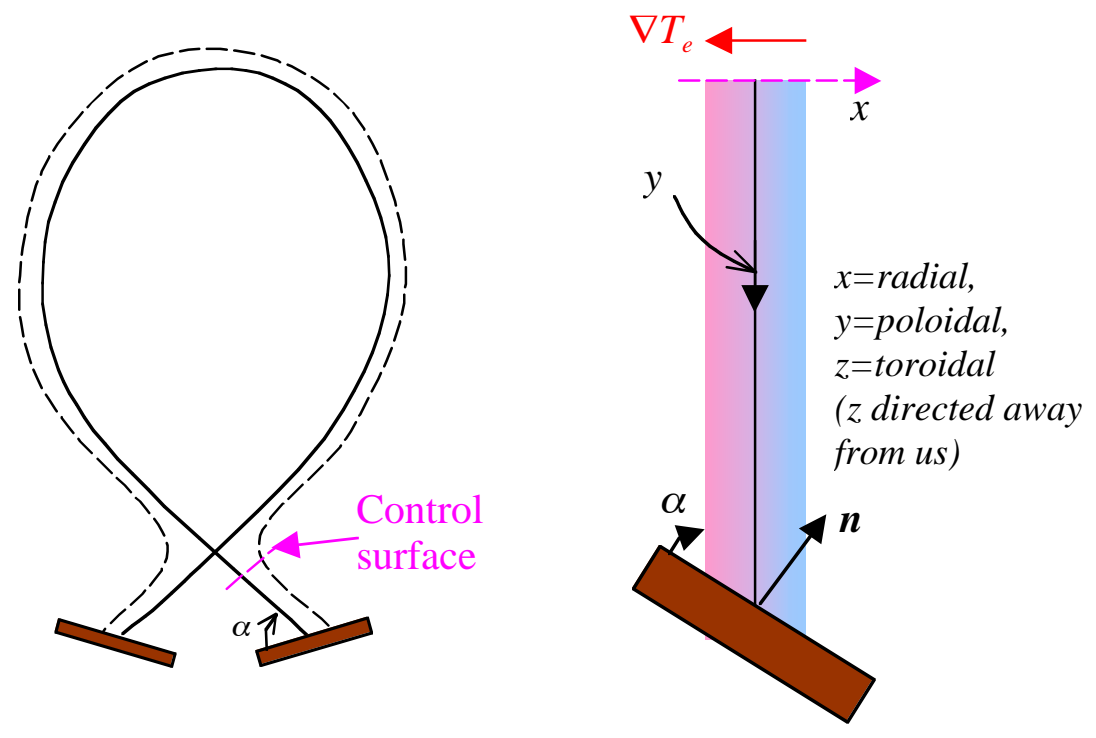

Fig. 1. a) The geometry of a tokamak with a poloidal divertor. Divertor legs are assumed to be long enough, so that exact location of the control surface near the x-point Is unimportant. b) An enlarged depiction of the divertor leg between the controlled surface (dashed line) and the divertor plate (a common flux region is shown; the separatrix is the left boundary of the plasma slab).

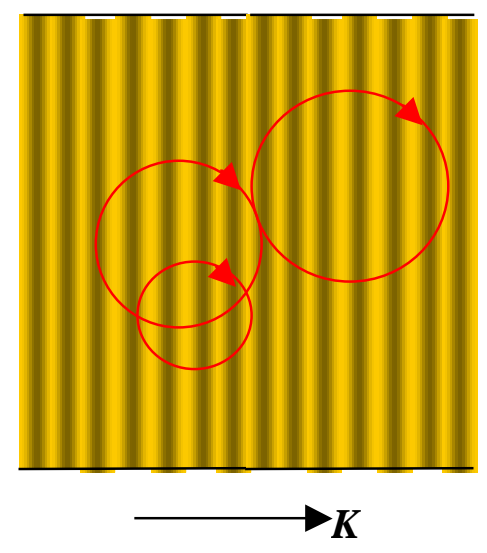

Fig. 2. The structure of the potential perturbation at some distance above the control surface, with a few ion gyro-orbits. The local wave vector $\boldsymbol{K}$ is directed almost perpendicularly to the flux surface and is larger than the inverse ion gyroradius. The average cross-velocity of the ions is therefore much less than $v_{\mathrm{D}}$. Electrons, on the other hand are magnetized. 


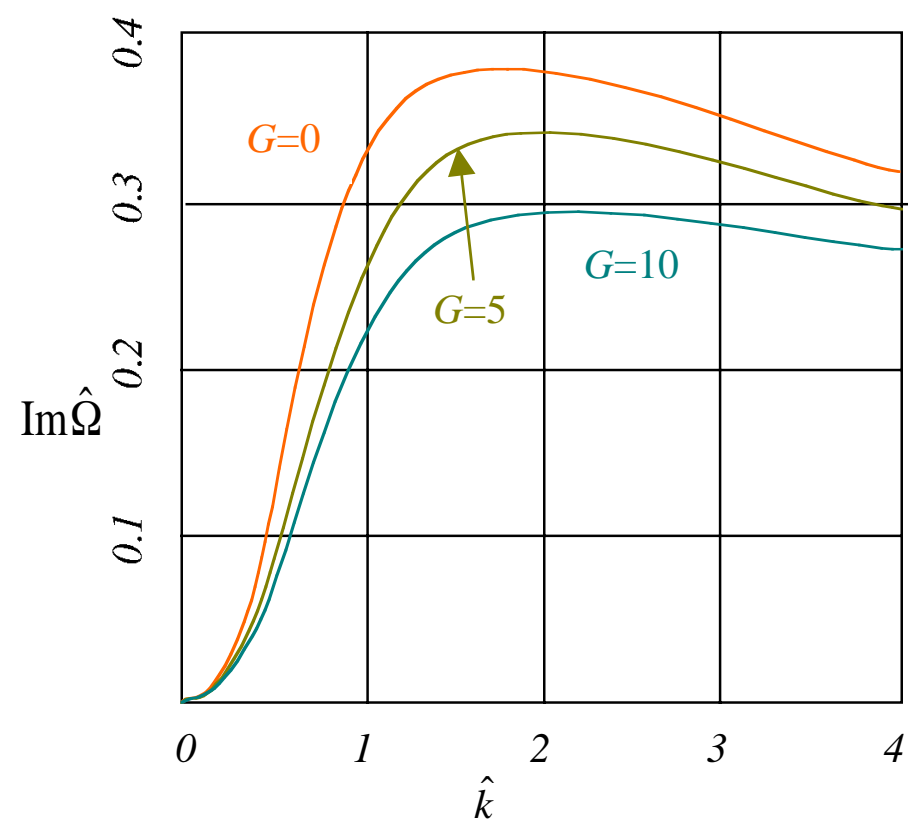

Fig.3. The dimensionless growth rate for $\alpha=0$ and various values of the parameter $G$.

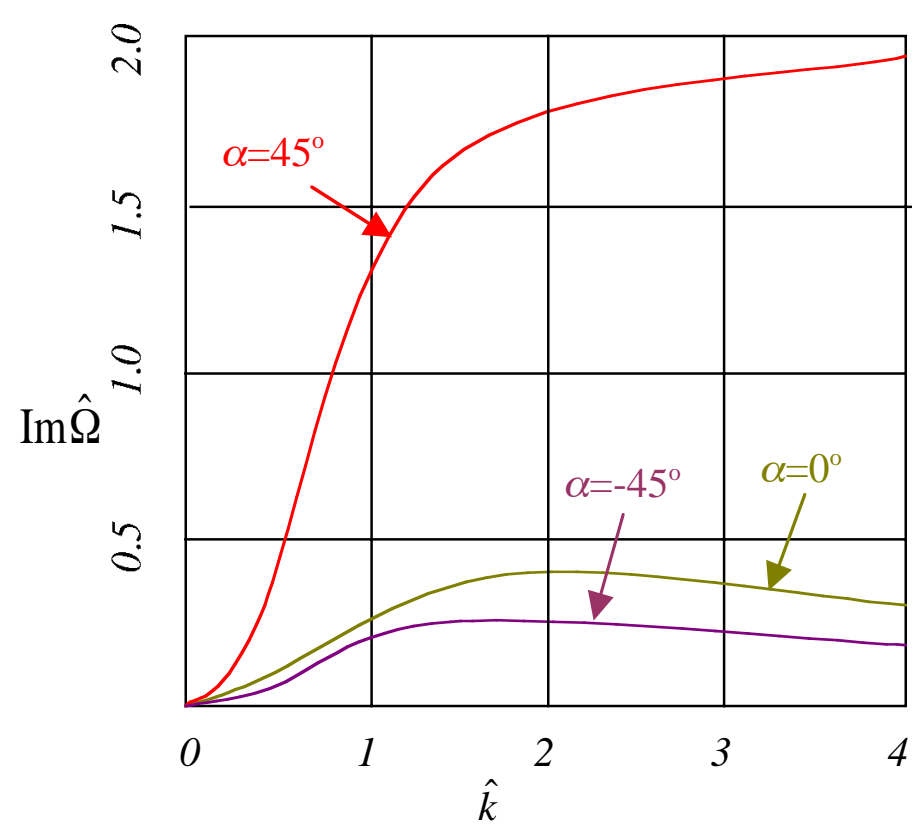

Fig.4. The dimensionless growth rate for various tilts of the divertor plate. The positive tilt corresponds to the situation shown on Fig. 1a. Parameter $G$ is equal to 5 in all cases. 